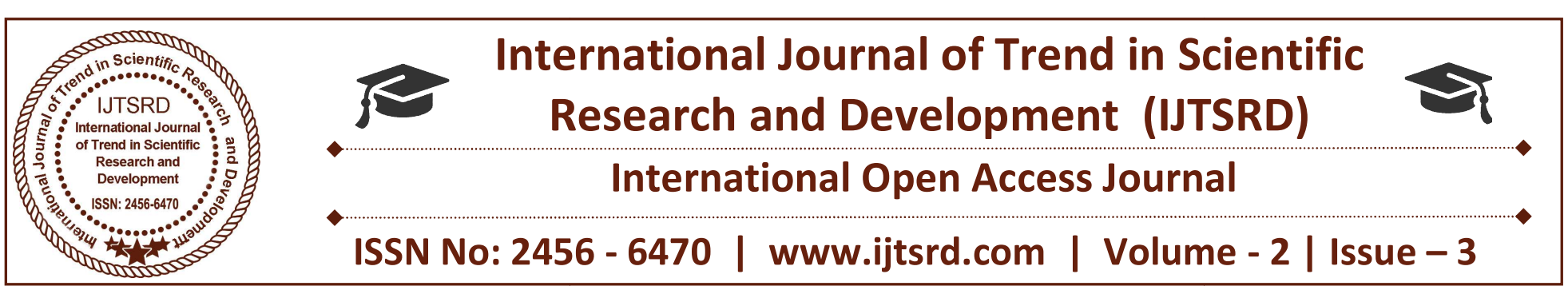

\title{
Alzheimer Patient Tracking and Alert System
}

\author{
Haroon Dar', Shreyansh Jain ${ }^{2}$, Jyoti Shukla ${ }^{3}$, V. M. Sardeshmukh ${ }^{4}$ \\ ${ }^{1,2,3}$ Student, Dept. of Electronics \& Telecommunication, Sinhgad Academy of Engineering, Pune, India \\ ${ }^{4}$ Professor, Dept. of Electronics \& Telecommunication, Sinhgad Academy of Engineering, Pune, India
}

\begin{abstract}
Locating and securing an Alzheimer's patient who is outdoors and in wandering is crucial to an Alzheimer patient safety. Although advances in geo tracking and mobile technology have made locating patients instantly possible, reaching them while in wandering state may take time. However, a social network of caregivers may help shorten the time that it takes to reach and secure a wandering $\mathrm{AD}$ patient. This study proposes a new type of intervention based on novel mobile application architecture to form and direct a social support network of caregivers for locating and securing wandering patients as soon as possible. System employs, aside from the conventional tracking mechanism, a wandering detection mechanism, both of which operates through a tracking device installed a Subscriber Identity Module for Global System for Mobile Communications Network (GSM).
\end{abstract}

Keywords: Alzheimer Disease (AD), Global System for Mobile Communication (GSM), Global Positioning System (GPS), Mild Cognitive Impairment (MCI), Electronic Geo- Tracking (EGT), Short Message Service (SMS), Radio Frequency Identification (RFID), Secure Digital Card (SD Card), Digital-to-Analog Converter (DAC), ActiveRadio Frequency Identification Localization System (ARFIDLS), General Packet Radio Service (GPRS), Real-time Locating Systems (RTLS).

\section{INTRODUCTION}

Alzheimer's Disease International estimates that more than 35 million people worldwide are living with Alzheimer's disease or a related dementia, and that number is expected to double in the next 20 years. Improving home and community-based coordinated care is critical to mitigating Alzheimer's effects on individuals and families and to reducing mounting healthcare costs.

Alzheimer's disease is the most common cause of dementia, and involves a progressive de generation of the cerebral cortex. There is widespread cortical atrophy. Neurons affected develop surrounding amyloidal plaques, neurofibrillary tangles, and produce less acetylcholine. The cause is not yet known. Patients experience irreversible global, progressive impairment of brain function, leading to reduced intellectual ability. It is estimated that 60 to $80 \%$ cases of dementia are Alzheimer's disease.[5]

About $40 \%$ of patients who have Alzheimer's disease get lost outside their homes during the course of their illness, presenting a substantial risk to their safety. Current methods of managing wandering behaviour include confinement, restraint, institutionalisation in care homes, and more There is no treatment still exist, early detection of $\mathrm{AD}$ still provide them and their families with an opportunity to plan for their future. In this paper, we are developing tracking and remainder system for Alzheimer's patient who are suffering from many difficulties such as memory loss that disturb daily life, challenging in planning or solving problems, difficulty completing familiar tasks, confusion with time and place, problems with words in speaking or writing.

The remainder of this paper is organized as follows. Section II described related work of present study. Section III describes proposed system of Alzheimer Patient tracking and alert system. Section IV presents results. Section V presents conclusion. 


\section{RELATED WORK}

The system presented in this article is designed to assist those who have mild dementia in overcoming problems such as memory loss that disturb daily life, challenging in planning or solving problems, difficulty completing familiar tasks, confusion with time and place, problems with words in speaking or writing. Our interest in this work was inspired by the study highlighting that approximately $40 \%$ of Alzheimer's patients who get lost outside their homes have substantial risk to their lives and due to which they have to face confinement, restraint, institutionalisation in care homes.

Various tracking and reminding deices have been designed in past.

One tracking system for AD patient was designed. Whenever AD patient has travelled outside then GSM in the caregiver initiates and received information from $\mathrm{AD}$ patient module. On the Visual Basic, the exact location of the AD patient has been seen. After every 1 minute GSM in AD patient module send message to the caregiver module. The exact coordinates of location and message sending is display on LCD. Whenever AD patients are wander around a city or anywhere then system is initiate and receive GPS co- ordinate of $\mathrm{AD}$ patient where he is wandering from GPS satellite. AD patient module is sending message after every 1 minute for informing caregiver AD patient is in safe zone or not.[5]

A Smart Mind an activity tracking and monitoring system targeted for patients with mild to moderate Alzheimer's symptoms was designed. The main sensor adopted in Smart- Mind is Kinect which captures the images and 3D posture data of the target (the patient) within his living environment. The tracked activity records can be reviewed by the patient's relatives and related medical professionals, e.g., community nurses, to assess his self-caring abilities. The apps called Smart-Reminder is a subsystem of Smart-Mind. Smart-Reminder performs four main functions to the target:

(1) people reminder; (2) item reminder; (3) activity reminder; and (4) mind training games.[3]

Another project was developed at Fraunhofer Portugal, the aim of this dissertation is to create a small electronic device able to check the environmental conditions in patients suffering from Alzheimer's disease (AD). This device is able to sense and monitor the environmental temperature and humidity, as well as the movements of the individual based on a free-fall detection system. The equipment can be incorporated into a piece of clothing (such as a waist belt). It is able to indicate the real-time position of the patient using GPS navigation and employs GSM-GPRS communication to transmit data to the caregiver and logging it into a database. A prototype has been developed and tested in patients from a retirement home.[2]

In another research, Alzheimer's Real Time Location System (ARTLS) was developed which is a localization system that can send Alzheimer's patient location feedback to caregiver in real time. The system is meant for monitoring the Alzheimer patient in real time to safeguard the patient's safety. The system is equipped with automatic implements recording the patient's movement sequence for caregiver references in enhancing the care management quality provided for the patient in the future. The recording resident movement data can be useful for the medical practitioners in studying the patient behavior and disease development based on movement sequence patterns shown by each resident.

Active-Radio Frequency Identification Localization System (ARFIDLS) is the reliable radio frequency localization system in enabling the RTLS. The RFID uses radio wave to transmit signal from the transponder (transmitter) or commonly known as tags to the RFID reader (Zhou \& Shi, 2009). The RFID allows us to identify objects or subjects with neither physical contact nor without line of sight. ARFIDLS is an ideal localization system in accommodating the RTLS for tracking the Alzheimer Disease. The major advantages of ARFIDLS are the nature of RFID that is automated. Identification or can be called as autoID (Cyplik \& Patecki, 2011), can detect many tagged items instantaneously, non-line of sight, wireless communication and wider coverage.[7]

A first generation mobile, video based reminder system was developed. The primary aim of the current project was to offer persons with mild $\mathrm{AD}$ an assistive presence in the home environment through the provision of frequent memory cues in the form of video reminders delivered via a mobile phone. Using a distributed Internet-based care model caregivers are provided with secure access to a custom interface through which video reminders can be recorded using an integrated we cam. Recorded reminders are scheduled by the caregiver and uploaded 
automatically via a secure connection to a backend database for future retrieval. At regular intervals, a collection of video reminders are transmitted via the server to the user's mobile phone where they are scheduled for playback, providing a virtual caregiver to assist with reminding users of daily activities.[8]

\section{PROPOSED WORK}

In this section, the detailing about the tracking and alert system of $\mathrm{AD}$ patient is given in the block diagram below:-

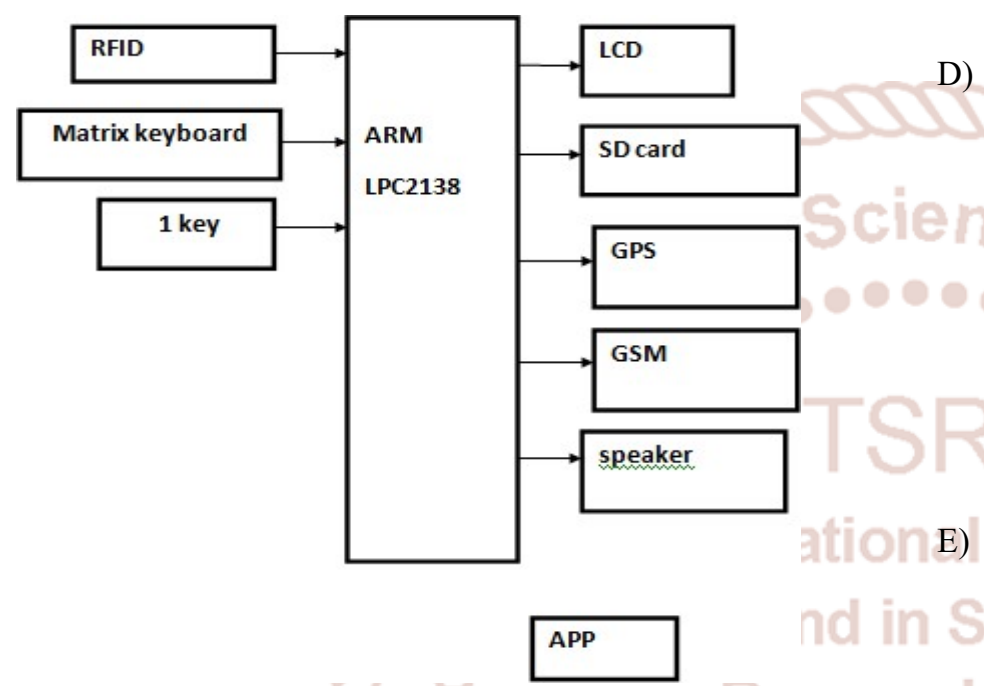

Fig (1): Block Diagram of Tracking and Alert System.

Here we are developing tracking and alert system for AD patients who suffer from many difficulties such as Memory loss that disrupts daily life, challenges in planning or solving problems, difficulty completing familiar task, confusion with time and place, problem with words in speaking or writing.

For this purpose we are developing a Alzheimer kit:

A) Memory Loss: Patient usually forget recently learned information. Even they can forget the address of their home. For this purpose, we are equipping them with a GPS tracking system, which will navigate the patient through the city.

B) Challenges in Planning: Some people may experience changes in their ability to develop and follow a plan or work with numbers. They may have trouble following a familiar recipe or keeping track of monthly bills. They may have difficulty concentrating and take things than they did before. For this purpose, we are developing a daily reminder system that will send SMS at regular intervals to remind the Alzheimer patient about the daily activities.

C) Difficulty completing familiar tasks: People with AD often find it hard to complete daily tasks. Sometimes, people may have trouble in completing a familiar task for such Bank based transaction. For this we are giving a Patient a RFID card through which he can do some base transactions. The RFID will contain the identity of the Patient which includes all his bank details and the manager of the bank will know the password of his Bank account.

D) Confusion with time and date: People with AD can lose track of dates, seasons and the passage of time. They may have trouble understanding something if it is not happening immediately. Sometime they may forget where they are or how they got there. For this reason, we are developing a GPS Tracking System and Reminder System which will continuously inform them about the date and time.

E) Problem with words in speaking or writing: People with AD may have trouble following or joining a conversation. They may stop in the middle of the conversation and have no idea how to continue or they may repeat themselves. For this reason, we are interfacing a SD card which can store wav audio files. The micro-controller reads this files and play them back using onboard DAC via speaker.

F) 1-Key(Emergency button): We are providing the kit with emergency button. If in case the patient is lost and if some serious issues happens to the patient in case if he is lost then the people around the patient will notice the emergency button $n$ will press it. After pressing this button the care-taker will get information about the patients location and the audio file will be generated via speaker which includes message (which will include name, address of the patient and it will inform the public that he/she is an AD patient ).

The AD patient module has ARM cortex M3 LPC1768, GPS antenna, power supply, 16*2 LCD display, and GSM and GPS module. The caregiver module has GSM module, PC and RS 232 kit. 


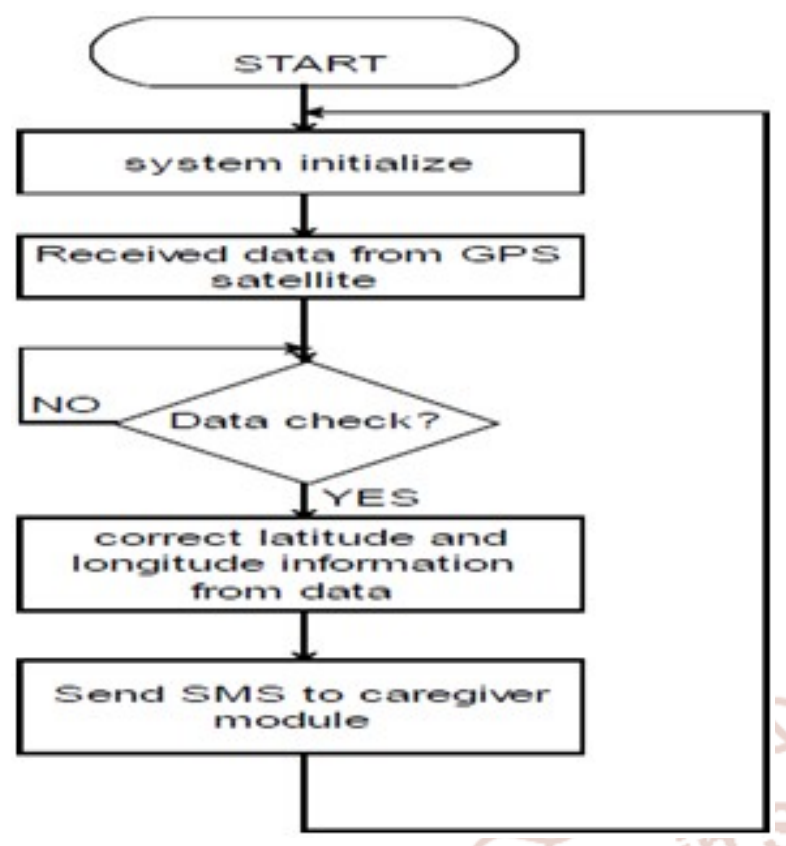

Fig (2): Flowchart of AD Patient module.

Whenever $\mathrm{AD}$ patients are wander around a city or anywhere then system is initiate and receive GPS coordinate of $\mathrm{AD}$ patient where he is wandering from GPS satellite. If receive date is correct then send GPS information to caregiver or otherwise it check coordinate again. AD patient module is sending message after every 1 minute for informing caregiver AD patient is in safe zone or not. Whenever AD patient has travelled outside then GSM in the caregiver initiates and received information from $\mathrm{AD}$ patient module.

\section{EXPECTED RESULT}

The result of the tracking system is shows the latitude and longitudinal co-ordinates and exact location of AD patient. From tracking system, caregiver is received the position of $\mathrm{AD}$ patient using GSM and GPS.

And the Emergency button, when pressed also gives us the exact location and wav a audio files via Speaker that tells a person about the patient's diseases.

\section{CONCLUSION}

From the above discussion, a detailed analysis of the various tracking systems has been performed, highlighting the technical shortcomings of the present methodology. It was seen how the various tracking systems have their own applications, strengths and also certain fallacies that lead to inaccurate results.
Also we providing a Alert system 1-key Emergency button for the patient who will suffer from AD so that caregiver can easily locate him when somebody presses it.

\section{Acknowledgement}

We would like to thank my all staff members of SAOE, Pune, who provided insight and expertise that greatly assisted the research. We want to specially thank our respected internal guide Prof. V. M. Sardeshmukh for his guidance and encouragement, which has helped us to achieve our goal. His valuable advice helped us complete our project successfully.

Our Head of Department Dr. M. M. Sardeshmukh has also been very helpful and we appreciate the support he provided us.

Last but not the least we would like to convey our gratitude to all the teaching and non-teaching staff members of Electronics and Telecommunication Department, our friends and families for their valuable suggestions and support.

\section{REFERENCES}

1. Alzheimer's Society. Alzheimer's society. http://alzheimers.org.uk/.

2. Priyanka Thakare, Dr. V.R.Pawar, "Alzheimer Disease detection and tracking of Alzheimer Patient," IEEE Journal of Biomedical and Health Informatics.

3. K.Lam, N.Tsang, "SmartMind: Activity Tracking and Monitoring for Patients with Alzheimers Disease" , IEEE proceeding, ICAINA, pp 453$460,2015$.

4. Y.Hsu, P. (Julia) Chung, M.Pai, "Gait and Balance Analysis for Patients with Alzheimers Disease Using an Inertial-Sensor-Based Wearable Instrument", IEEE Journal Of Biomedical And Health Informatics, Vol.18, No. 6, pp 1822-1830, November 2014. 
5. Pradyumna Shenvi, Pulkit Baheria, Shan Jose, Sachit Kumar, Jyothi S.Nayak,"Wearable Tracking device for Alzheimer's Patients: A Survey" JETIR volume 3, Issue 4, April 2016. http://www.jetir.org.

6. Lancer Neurology, "keep tracking of patient with Alzheimer disease", http://www.thelancet.com

7. Ana Rita Cardosod eAlmeida Barreto," Environment-Aware System for Alzheimer's Patients", FOR JURY EVALUATION,Mestrado Integrado em Engenharia Electrotécnica e de Computadores,Supervisors: Artur Cardoso (FEUP) Cândido Duarte (FEUP) Renato Oliveira: (Fraunhofer AICOS)

8. Mark Donnelly, Chris Nugent, Sally McClean, and Bryan Scotney, Sarah Maso,Peter Passmore and David Craig,"A Mobile Multimedia Technology to Aid Those with Alzheimer's Diseasen", University of Ulster, Belfast Trust Queen's University Belfast,1070-986X/10/\$26.00 c 2010 IEEE, Published by the IEEE Computer Society. 